\title{
Quantitative interpretation of the excitonic splittings in aluminum nitride
}

\author{
B. Gil ${ }^{1, a}$, B. Guizal ${ }^{1}$, D. Felbacq ${ }^{1}$, and G. Bouchitté ${ }^{2}$ \\ ${ }^{1}$ Groupe d'Étude des Semiconducteurs, UMR CNRS 5650, Université Montpellier 2, Case courrier 074, \\ 34095 Montpellier Cedex 5, France \\ 2 Institut de Mathématiques de Toulon, Université de Toulon et du Var, 83957 La Garde Cedex, France
}

Received: 5 November 2010 / Accepted: 9 November 2010

Published online: 28 January 2011 - (C) EDP Sciences

\begin{abstract}
We address the interpretation of the splitting between the ground state excitonic transition which indicates the energy of the lowest direct band gap in AlN bulk films and epilayers, and a 36-38 meV higher energy companion. We demonstrate that this splitting is consistent with the initial interpretation in terms of $1 s-2 s$ excitonic splitting by using a calculation of the exciton binding energy which includes mass anisotropy and anisotropy of the dielectric constant. Analytical expressions are proposed to compute the evolution of $1 s$ and $2 s$ excitonic energies using an anisotropy parameter. We show that the values of the dielectric constant that are required to fit the data are $\varepsilon_{\perp} \approx 8.7$ and $\varepsilon_{\|} \approx 10$, values different from the couple of values $\varepsilon_{\perp} \approx 7.33$ and $\varepsilon_{\|} \approx 8.45$ erroneously obtained after a fitting procedure using a spherical description of the long range Coulomb interaction and the classical textbook $n^{-2}$ spectrum of the excitonic eigenstates. Starting from now, our values are the recommended ones.
\end{abstract}

\section{Introduction}

Aluminum nitride is an interesting material for realizing light emitting or light detecting devices susceptible to operate in the upper UVB energy region [1]. The optical properties of bulk aluminum nitride and of its epilayers are currently under very intense investigations [2]. The crystal field splitting of bulk AlN has been identified as being negative contrarily to the $\mathrm{GaN}, \mathrm{InN}$ and $\mathrm{CdS}$ case [3], which gives an interesting and well marked anisotropy of the optical response of this material. The optical properties of epilayers are in addition modified in relation with builtin strain fields and experimental values of the excitonic deformation potentials were recently proposed [4-7]. The evolution of the optical properties of AlN epilayers grown on various substrates are now well accounted for by models which were fruitfully applied to $\mathrm{GaN}$ [8], $\mathrm{ZnO}$ [9]. Figure 1 is for AlN, the analog of figure 10 of reference [10] dedicated to GaN. It indicates that both growth of high quality AlN and interpretation of strain effects are now achieved. It also indicates that the valence band splitting is always large compared to the value of the excitonic binding energy, a situation which validates the one-band calculation below.

\section{Experimental values of the excitonic splitting}

Silveira et al. [11] have reported reflectance structures at $6.029 \mathrm{eV}$ and $6.065 \mathrm{eV}$ at $6 \mathrm{~K}$. They attributed this $36 \mathrm{meV}$

\footnotetext{
a e-mail: bernard.gil@univ-montp2.fr
}

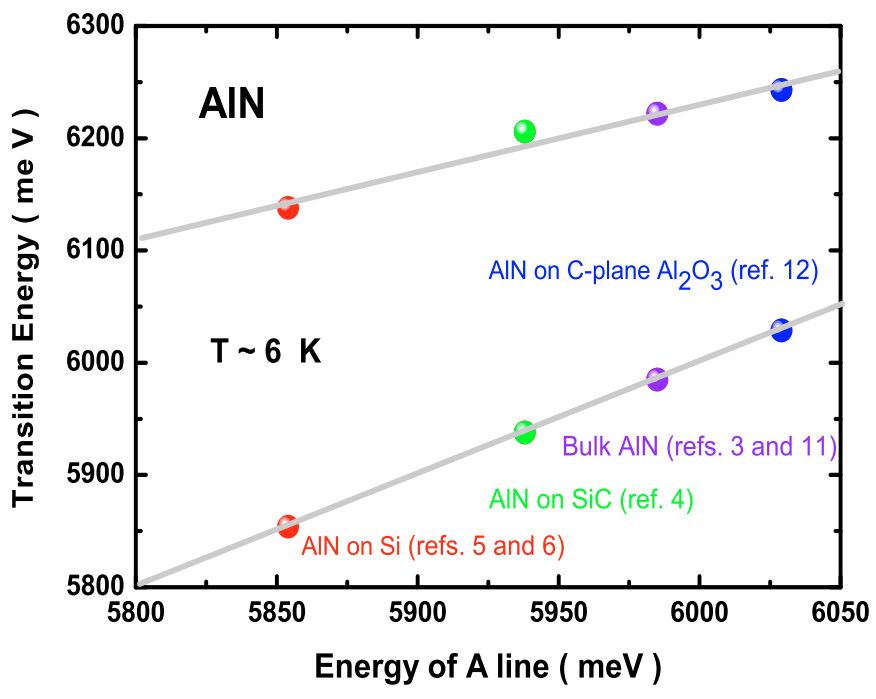

Fig. 1. (Color online) Plot of the excitonic energies in various AlN bulk materials and epilayers grown on foreign substrates versus energy of line A. The fit to the data is coherent with the values of deformation potentials in reference [7]. The data of reference [6] taken at $300 \mathrm{~K}$ was blue shifted of $65 \mathrm{meV}$ to match the $6 \mathrm{~K}$ data.

splitting to $1 s-2 s$ splitting. By straightforward application of the physics of hydrogenic atoms they concluded the exciton binding energy to be $48 \mathrm{meV}$. This would locate the band gap $E_{g}$ of unstrained AlN at $\sim 6.080 \mathrm{eV}$ at low temperature. Similar energy splitting was reported by Feneberg et al. [2]. Considering the strained layer epitaxy 
and the report of Onuma et al. [12], we note a $38 \mathrm{meV}$ splitting after processing of their data. Still using the model of the hydrogen atom, though slightly doubting about its applicability (see row 30, first column of page 023529-6 of Ref. [12]), they however report an exciton binding energy of $52 \mathrm{meV}$ after giving the ad hoc value to the dielectric constant. Yamada et al. [13] have reported $(1 s)$ free exciton photoluminescence at $6.0346 \mathrm{eV}$ and a $2 s$ related one at $6.0772 \mathrm{eV}$. The experiment was carried out at $77 \mathrm{~K}$, which is not a problem now that we know very well the temperature dependence of the band gap of AlN [2]. The $1 s$ photoluminescence peak is indicated at an energy that we estimated some $\sim 6 \mathrm{meV}$ lower than the energy of the transverse exciton deduced from a line shape fitting of the reflectance feature given with the photoluminescence data in figure 1 of reference [13]. This blue shift is due to thermalization effects at the bottleneck of the polariton dispersion relation. This is a very well established effect in semiconductors in general [14] and in nitride in particular $[2,15]$. The large value of the oscillator strength (and of the longitudinal transverse splitting) in AlN [2] is responsible of this substantial shift. The smaller value of the longitudinal transverse splitting (or said using another phrasing of the oscillator strength) of the $2 s$ exciton does not give so marked thermalization at the bottle neck of the $2 s$ exciton polariton. Therefore the $6.0772 \mathrm{eV}$ value at the maximum of the $2 s$ photoluminescence peak is almost the energy of the $2 s$ transverse exciton polariton. This analysis leads us to conclude that the $1 s-2 s$ splitting is about $37 \mathrm{meV}$ in this sample.

It is worthwhile noticing that the excitonic fine structure splitting [16] has not been discussed here: the shortrange spin exchange interaction may equal a few meV [2]. Finally we consider having another uncertainty arising from various inhomogeneous broadening effects in the experimental reports leading to a $\sim 1 \mathrm{meV}$ scattering in the determination of this splitting. The optical properties of anisotropic semiconductors like CdS, GaSe, $\mathrm{MoS}_{2}$ to be clearly interpreted required using an excitonic model which includes anisotropy effects on mass and dielectric constant [17]. We have decided to treat the AlN case by also including mass anisotropy effects in the one hand and to offer our readers simple analytical expressions susceptible to correlate the energy levels and the excitonic oscillator strengths with the anisotropy of the band structure in another hand.

\section{Model}

The solutions of the exciton problem in an isotropic semiconductor are the well-known hydrogen like functions $\Phi^{n l m}(r)$, and the eigenvalues are given by $E_{\text {exc }}^{n l m}=$ $E_{g}-R / n^{2}$ where: $R=2 m_{0} c^{2} \alpha^{2} \frac{\mu}{\varepsilon^{2}}$. In the preceding formula, $\alpha$ is the fine structure constant which characterizes the strength of the electromagnetic interaction, $m_{0}$ is the electron mass at rest and $c$ is the in vacuum velocity of light, $\mu$ is the isotropic electron-hole reduced mass and $\varepsilon$ is the isotropic dielectric constant of the medium where electron and holes orbit with respect to each other. $R$ is an ef- fective Rydberg energy. The calculation of exciton binding energies in anisotropic semiconductors is a complicated mathematical problem [17]. There is no longer an analytical solution to that problem when anisotropy occurs in the kinetic energy term or in the potential energy term of the Schrödinger equation used to describe the exciton in anisotropic media. Exciton states $\left|\Psi^{\beta}\right\rangle$ are classified in accordance with the irreducible representations of the cylindrical group $D_{\infty}^{h}$.

Exciton effects in crystals which are anisotropic along a crystal axis ( $z$-axis) to be treated require to introduce the reduced masses parallel and perpendicular to the direction of the $z$-axis, $\mu_{\|}$and $\mu_{\perp}$ and the corresponding dielectric constants $\varepsilon_{\|}$and $\varepsilon_{\perp}$ and one defines an anisotropy parameter $\gamma=\frac{\varepsilon_{\perp}}{\varepsilon_{\|}} \frac{\mu_{\|}}{\mu_{\perp}}$ which rules the eigenstates and eigenvectors spectra $[7,17-19]$.

When $\gamma=1$, the ground state is purely the three dimensional $1 s$ state. When $\gamma=0$, we face two-dimensional analytical solutions. For other values of $\gamma$ levels are $\gamma$-dependent admixtures of $1 s, 2 s, 3 s, 3 d(m=0), 4 s, \ldots$ The mathematical calculation of the eigenstates, that is to say calculation of the excitonic binding energies, and their eigenstates are here proposed by using the formalism of hyperspherical harmonics [18] which is very well adapted to treat the long range interaction in anisotropic crystals [19].

Besides the technical aspects of the calculation which are extremely interesting from the mathematical point of view, it is important to emphasize that the ground state is given with a very good accuracy in units of the reduced Rydberg energy: $R^{*}=2 m_{0} c^{2} \alpha^{2} \frac{\mu_{\perp}}{\varepsilon_{\|} \varepsilon_{\perp}}$, by the simple equation:

$$
E_{1 S} \approx-\frac{4}{\left(1+\gamma^{\frac{1}{3}}\right)^{2}}
$$

Note here the $\gamma^{\frac{1}{3}}$ dependence [19]. Similarly, a simple fitting procedure of the evolution of the $2 s$ state enables us to propose the more complicated although still quite simple following behavior:

$$
E_{1 s-2 s} \approx-0.47-\frac{200}{150+27 \mathrm{e}^{-3 \gamma^{\frac{1}{3}}}} .
$$

These simple equations are no longer obtained for higher index states due to complicated anti-crossing behaviors and the labeling of the eigenstates using the $n l m$ series of hydrogenic quantum numbers then becomes meaningless. This is not the case for the $1 s$ and $2 s$ states which are fairly decoupled from the other levels and for which this labeling can be reasonably kept.

In Figure 2 has been plotted the evolution of $1 s-2 s$ splitting relative to the $1 s$ exciton binding energy as a function of the anisotropy parameter $\gamma$.

It is worthwhile noticing that the ratio between the oscillator strengths of $1 s$ and $2 s$ states can also be expressed using a simple expression:

$$
\frac{\Psi_{1 s}^{2}(0)}{\Psi_{2 s}^{2}(0)} \approx 15 \frac{\gamma^{\frac{1}{3}}}{2 \gamma^{\frac{1}{3}}-0.13} .
$$




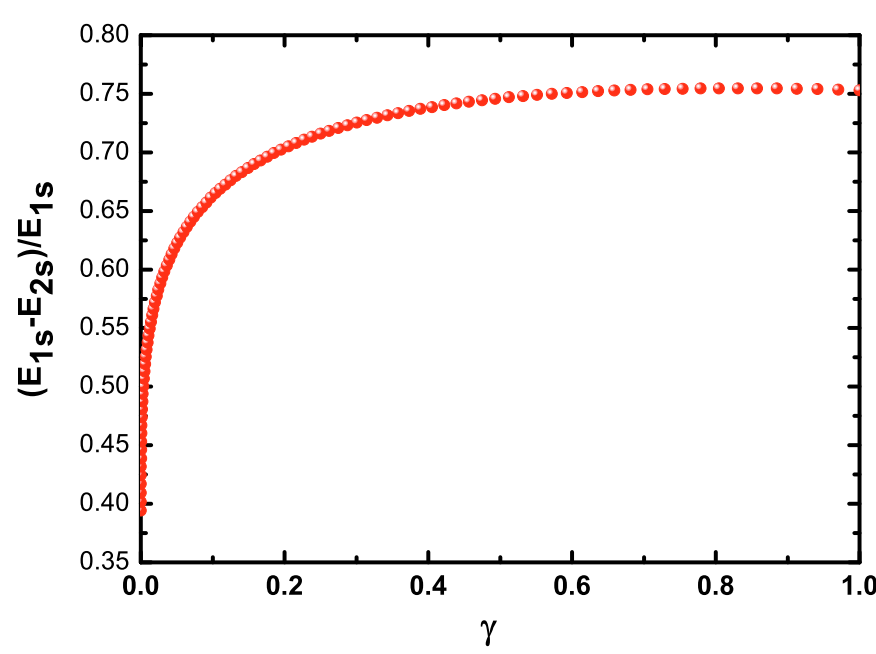

Fig. 2. (Color online) Plot of the $1 s-2 s$ splitting relative to the $1 s$ exciton binding energy versus anisotropy parameter $\gamma$.

Table 1. Values of Lüttinger parameters and electrons masses proposed in the AlN-related literature.

\begin{tabular}{ccccc}
\hline$\left|A_{1}\right|$ & $\left|A_{2}\right|$ & $m_{e \|}$ & $m_{e \perp}$ & Reference \\
\hline 4.06 & 0.26 & 0.33 & 0.25 & {$[20]$} \\
3.82 & 0.22 & 0.32 & 0.33 & {$[21]$} \\
3.85 & 0.25 & 0.35 & 0.35 & {$[22]$} \\
4.367 & 0.518 & 0.25 & 0.24 & {$[23]$} \\
4.46 & 0.25 & 0.30 & 0.29 & {$[24]$} \\
3.991 & 0.311 & 0.322 & 0.329 & {$[25]$} \\
\hline
\end{tabular}

This ratio does not significantly deviate from the value in spherical symmetry (8) until significant anisotropies are reached, that is to say for $\gamma<0.4$.

\section{Comparison with experiments}

This study has offered us the opportunity to propose analytical dependences of the $1 s$ and $2 s$ excitonic binding energies with anisotropy parameter $\gamma$ and an analytical dependence of their oscillator strength ratio. With the help of these analytical expressions one now may get rid of making sophisticated mathematical calculations which constitutes a considerable saving of time for experimentalists. We now have to compute the exciton in case of the AlN semiconductor for which conduction and valence band dispersion relations have been computed by several groups [20-25] using first principle calculation approaches. The anisotropy of hole masses can be represented in the framework of the k.p theory $[7,26]$.

The fundamental valence band of AlN has $\Gamma_{7}$ symmetry [3], its wave functions is mainly built from $\left|\Gamma_{7}^{2}\right\rangle\left(p_{z}\right.$ type Bloch waves), the hole effective mass are $\approx\left|\frac{1}{A_{1}}\right|$ and $\approx\left|\frac{1}{A_{2}}\right|$ along the [001] axis and in the orthogonal direction respectively. In terms of excitonic masses one obtains [7]: $\frac{1}{\mu_{\|}} \approx \frac{1}{m_{e} \|}+\left|A_{1}\right|$, and $\frac{1}{\mu_{\perp}} \approx \frac{1}{m_{e \perp}}+\left|A_{2}\right|$.

Table 1 summarizes the values of the effective masses and Lüttinger parameters obtained by different groups in

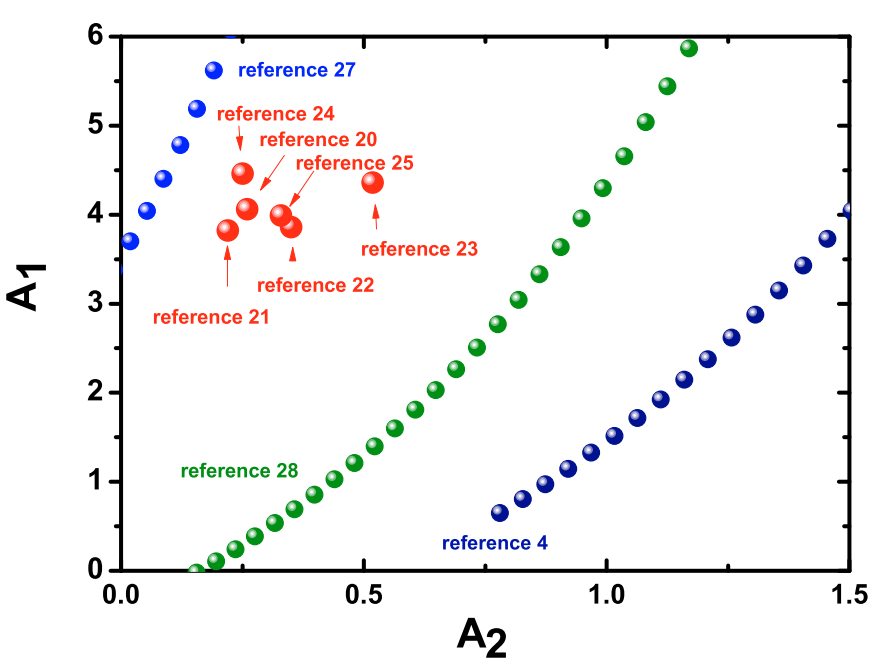

Fig. 3. (Color online) Plot of the theoretical values of ( $A 1$, $A 2)$ couples in AlN (large red dots). Plots of the values of these $c$ couples in agreement with a $38 \mathrm{meV}$ splitting between $1 s$ and $2 s$ excitons, computed using different values of the AlN dielectric constant (blue, olive or navy series of dots).

Table 2. Values of dielectric constants proposed in the AlNrelated literature.

\begin{tabular}{ccc}
\hline$\varepsilon_{\perp}$ & $\varepsilon_{\|}$ & Reference \\
\hline 9.06 & 10.44 & {$[27]$} \\
7.76 & 9.32 & {$[28]$} \\
7.33 & 8.45 & {$[4]$} \\
\hline
\end{tabular}

the context of sophisticated first principle calculations. All groups obtain comparable results except reference [23], which we do not understand.

The situation is much more complicated concerning values of the dielectric constants as shown in Table 2. Facing the difficulty to choose a given pair of values we have decided to plot the values of the $A_{1}$ and $A_{2}$ Lüttinger parameters compatible with an experimental value of $38 \mathrm{meV}$ to the $1 s-2 s$ splitting using our mathematical treatment which has given us the dimensionless value $E_{1 s-2 s}$ of this splitting as analytical functions of the anisotropy parameter $\gamma$ and of the reduced Rydberg energy. After some simple algebraic manipulation, one gets:

$$
\left|A_{1}\right|=2 m_{0} c^{2} \alpha^{2} \frac{E_{1 s-2 s}}{\gamma \Sigma \varepsilon_{\|}^{2}}-\frac{1}{m_{e \|}}
$$

and

$$
\left|A_{2}\right|=2 m_{0} c^{2} \alpha^{2} \frac{E_{1 s-2 s}}{\Sigma \varepsilon_{\|} \varepsilon_{\perp}}-\frac{1}{m_{e \perp}} .
$$

In Figure 3 are plotted versus $A_{2}$, the values of $A_{1}$ compatible with $\Sigma=38 \mathrm{meV}$ obtained using electron masses $m_{e \|}=0.32$ and $m_{e \perp}=0.28$, which are "average" theoretical values [11] (their choice is not impacting very much the rest of this paper in fact) using the different couples of the dielectric constant $[4,27,28]$ given in Table 2 .

The theoretical values of the Lüttinger parameters are represented using red dots. Obviously the agreement between the theoretical values and the experiment requires 
using substantially high values of the dielectric constant. We came to the conclusion that the best agreement between the theoretical values of the valence and conduction band dispersion relation and the experimental splitting requires values of the dielectric constant that are typically $\varepsilon_{\perp} \approx 8.7$ and $\varepsilon_{\|} \approx 10$, values which are in the range of experimentally reported ones. This gives an anisotropy parameter $\gamma=0.47$, a reduced Rydberg energy of $40 \mathrm{meV}$ and a $1 s$ excitonic binding energy of $51 \mathrm{meV}$. It is worthwhile emphasizing here is that the $1 s-2 s$ splitting is about 0.747 times the excitonic binding energy as seen in Figure 2 , a value close to the value for the spherical situation; 0.75 . However we remind that the excitonic binding energy is $\sim 1.27$ times the value of the reduced Rydberg energy: $R^{*}=2 m_{0} c^{2} \alpha^{2} \frac{\mu_{\perp}}{\varepsilon_{\|} \varepsilon_{\perp}}$.

Note that the value of the ratio $\varepsilon_{\perp} / \varepsilon_{\|}$equals 0.87 . According to reference [4], 0.868 appears to be the value the most compatible with experimental reports, compared to the alternate 0.833 value. There o many digits in these ratio and using one or another just leads to a non impacting 4 percent change in $\gamma$. We followed reference [4] and decided to take ratio 0.87 . This decision does not impact very much the message in this article.

\section{Conclusion}

By using a model of the excitonic energies that includes both mass and dielectric constant anisotropies we could account for the excitonic splitting of AlN. Using for band structure inputs the average theoretical values $m_{e \|}=0.32$, $m_{e \perp}=0.28, A_{1}=-3.95$ and $A_{2}=-0.27$ we can bring the agreement between the calculation and the experiment using for the dielectric constants values $\varepsilon_{\perp} \approx 8.67$, and $\varepsilon_{\|} \approx 10$ which are in the range of experimental findings. This in depth investigation establishes that exciton binding energy associated to the lowest band gap unambiguously equals $51 \mathrm{meV}$ in AlN.

B. Gil and G. Bouchitté acknowledge the financial support of CNRS under contract "PEPS ANISEXCIT".

\section{References}

1. T. Onuma, K. Hazu, A. Uedono, T. Sota, S.F. Chichibu, Appl. Phys. Lett. 96, 061906 (2010)

2. M. Feneberg, R.A.R. Leute, B. Neuschl, K. Thonke, Phys. Rev. B 82, 075208 (2010)

3. L. Chen, B.J. Skromme, R.F. Dalmau, R. Schlesser, Z. Sitar, C. Chen, W. Sun, J. Yang, M.A. Khan, M.L. Nakarmi, J.Y. Lin, H.-X. Jiang, Appl. Phys. Lett. 85, 4334 (2004)
4. H. Ikeda, T. Okamura, K. Matsukawa, T. Sota, M. Sugawara, T. Hoshi, P. Cantu, R. Sharma, J.F. Kaeding, S. Keller, U.K. Mishra, K. Kosaka, K. Asai, S. Sumiya, T. Shibata, M. Tanaka, J.S. Speck, S.P. DenBaars, S. Nakamura, T. Koyama, T. Onuma, S.F. Chichibu, J. Appl. Phys. 102, 123707 (2007)

5. G.M. Prinz, A. Ladenburger, M. Schirra, M. Feneberg, K. Thonke, R. Sauer, Y. Taniyasu, M. Kasu, T. Makimoto, J. Appl. Phys. 101, 023511 (2007)

6. G. Rossbach, M. Rösspischer, P. Schley, G. Gobsch, C. Werner, C. Cobet, N. Esser, A. Dadgar, M. Wienecke, A. Krost, R. Goldhahn, Phys. Stat. Sol. (b) 247, 1679 (2010)

7. B. Gil, Phys. Rev. B 81, 205201 (2010)

8. B. Gil, O. Briot, R.L. Aulombard, Phys. Rev. B 52, R17028 (1995)

9. B. Gil, A. Lusson, V. Sallet, S.-A. Said-Hassani, R. Triboulet, P. Bigenwald, Jpn J. Appl. Phys. Lett. 40, L1089 (2001)

10. B. Gil, in Stress effects on optical properties, Gallium nitride $(G A N)$, Vol. II, edited by J.I. Pankove, T.D. Moutsakas (Academic Press, New York, 1999), pp. 209274

11. E. Silveira, J.A. Freitas Jr., O. Glembocki, G.A. Slack, L.J. Schowalter, Phys. Rev. B 71, 041201 (2005)

12. T. Onuma, T. Shibata, K. Kosaka, K. Asai, S. Sumiya, M. Tanaka, T. Sota, A. Uedono, S.F. Chichibu, J. Appl. Phys. 105, 023529 (2009)

13. Y. Yamada, K. Choi, S. Shin, H. Murotani, T. Taguchi, N. Okada, H. Amano, Appl. Phys. Lett. 92, 131912 (2005)

14. J.J. Hopfield, Phys. Rev. 112, 1555 (1958)

15. B. Gil, S. Clur, O. Briot, Solid State Commun. 104, 267 (1997)

16. B. Gil, O. Briot, Phys. Rev. B 55, 2530 (1997)

17. A. Baldereschi, M.G. Diaz, Nuovo Cimento B 68, 217 (1970)

18. V.A. Fock, Z. Phys. 98, 145 (1935)

19. E.A. Muljarov, A.L. Yablonskii, S.G. Tikhodeev, A.E. Bulatov, J.L. Birman, J. Math. Phys. 41, 6026 (2000)

20. M. Suzuki, T. Uenoyama, A. Yanase, Phys. Rev. B 52, $8132(1995)$

21. J.A. Majewski, M. Städele, P. Vogl, MRS Internet J. Nitride Semicond. Res. 1, 30 (1996)

22. K. Kim, W.R.L. Lambrecht, B. Segall, M. van Schilfgaarde, Phys. Rev. B 56, 7363 (1997)

23. D.J. Dugdale, R. Brand, R. Abram, Phys. Rev. B 61, 12933 (2000)

24. C. Persson, Bo E. Sernelius, A. Ferreira da Silva, R. Ahuja, B. Johansson, J. Phys.: Condens. Matter 13, 8915 (2001)

25. P. Rinke, M. Winkelnkemper, A. Qteish, D. Bimberg, J. Neugebauer, M. Scheffler, Phys. Rev. B 77, 075202 (2008)

26. M. Kumagai, S.L. Chuang, H. Ando, Phys. Rev. B 57, 15303 (1998)

27. J.G. Tischler, J.A. Freitas Jr., Appl. Phys. Lett. 85, 1943 (2004)

28. W.J. Moore, J.A. Freitas Jr., R.T. Holm, O. Kovalenkov, V. Dmitriev, Appl. Phys. Lett. 86, 141912 (2005) 